\title{
SURFACE RECONSTRUCTION FOR GENERATING DIGITAL MODELS OF PROSTHESIS
}

\author{
Luiz C. M. de Aquino, Diego A. T. Q. Leite, Gilson A. Giraldi \\ National Laboratory for Scientific Computing, Petrópolis, Brazil \\ lcaquino@lncc.br,diegoamim@gmail.com,gilson@lncc.br \\ Jaime S. Cardoso \\ INESC Porto, Faculty of Engineering, University of Porto, Porto, Portugal \\ jaime.cardoso@ieee.org \\ Paulo Sergio S. Rodrigues ${ }^{1}$ and Luiz A. P. Neves ${ }^{2}$ \\ FEI ${ }^{1}$, São Bernardo do Campo, Brazil; and Federal University of Paraná ${ }^{2}$, Brazil \\ psergio@fei.edu.br,neves@ufpr.br
}

Keywords: Digital prosthesis design; segmentation; Deformable model, shape recovery.

\begin{abstract}
The restoration and recovery of a defective skull can be performed through operative techniques to implant a customized prosthesis. Recently, image processing and surface reconstruction methods have been used for digital prosthesis design. In this paper we present a framework for prosthesis modeling. Firstly, we take the computed tomography (CT) of the skull and perform bone segmentation by thresholding. The obtained binary volume is processed by morphological operators, frame-by-frame, to get the inner and outer boundaries of the bone. These curves are used to initialize a $2 D$ deformable model that generates the prosthesis boundary in each CT frame. In this way, we can fill the prosthesis volume which is the input for a marching cubes technique that computes the digital model of the target geometry. In the experimental results we demonstrate the potential of our technique and compare it with a related one.
\end{abstract}

\section{INTRODUCTION}

The repairing of a defective skull is needed not only for aesthetic factors but also because large defects in the skull may expose a significant area of the brain. In this case cranioplasty (the procedure of repairing holes in the skull with cranial implants) becomes necessary (Sanan and Haines, 1997). In traditional surgical procedures, cranioplastic implants were manufactured directly in the operating-theater where the surgeon modeled by hand the material, namely polymethylmethacrylate (PMMA), to make the prosthesis. However, the success of such procedure depends strongly on the manual skill of the surgeon, with a high risk of infection and a certain percentage of failure due to rejection (Solaro et al., 2008).

Nowadays cranioplasty continues to be a challenge to craniofacial surgeons which motivates the development of computational tools for surgical planning and implants design (Chen et al., 2006; Lee et al., 2009; Solaro et al., 2008).

In early 1980s, the introduction of 3-D CT scans and surface reconstruction methods provided a new effective tool for surgical planning. The generated digital models and stereolithography rapid prototyp- ing technologies (RP) made skull prostheses manufacture more accurate and customized for each case (Lee et al., 2002).

The application of medical image processing, computer graphics and rapid prototyping (RP) technology allows the customization of cranioplasty procedures to relieve the injury of patients in operations (Kai et al., 2009). The pipeline for the prosthesis generation begins with the acquisition of the computed tomography (CT) image of the skull. Then, image processing methods are applied for bone segmentation. Next, some surface reconstruction method is applied to get a three-dimensional model of the prosthesis. Such model is the input for the next step where the prosthesis of defective skull is manufactured using RP technology. Lastly, the prosthesis is implanted into the defective position.

From the viewpoint of image processing and geometric modeling the approaches in this area fall into two main categories. In the first one surface reconstruction is usually applied to get a representation of the defective skull which is then used to reconstruct the digital model of the lost part. In (You et al., 2009) we find one of such method, which divides each voxel into tetrahedra and applies a marching technique to 
get a polygonal representation of the defective skull. Then, the method uses user-defined guiding points and computational geometry algorithms to generate the digital version of the prosthesis taking into account only the polygonal mesh.

The second class of techniques includes methods that first reconstruct the skull in the image space. Then, they apply Boolean operations and Marching Cubes methods to get the digital model of the prosthesis. Reflection techniques, based on the assumption of skull symmetry, belong to this class. In this case, we can compute the axis of symmetry and reflect the segmented image (bone) respect to this axis. However, this method can be used only if the lesion do not cross that axis.

In this paper, we propose a new methodology for digital prosthesis generation, which falls into the second category. Our proposal is roughly divided into 5 stages: 1) Slice-by-Slice Segmentation: Extract the bone from the other tissues; 2) Feature Extraction: get geometric information about the frontier of the lesion (terminal points and their tangents); 3) Deformable Model: With the information from step 2, compute the inner and outer boundaries of the prosthesis, using a deformable $2 D$ model of type balloon; 4) Prosthesis Volume: Fill each patch obtained in the step above to complete the prosthesis volume; 5) Surface Reconstruction: Marching cubes is applied to generate the digital version of the prosthesis geometry.

To accomplish the first step, we take each frame of the volume and we apply a simple thresholding technique based on image inspection. Then, we perform the feature extraction. Firstly, we use an edge detector to extract the boundary $B$ of the bone in the binary image and a thinning process to get the skeleton of the bone. Then, the convex hull $S$ is computed for the skeleton. We consider the difference $B-S$ as the outer boundary of the bone and the set $B \cap S$ as the inner boundary of the bone. The obtained curves are used to get the geometric information need for the next step. We take the end points and tangents as boundary conditions for a deformable model, a balloon-like one (Cohen, 1991), which is used to compute the patch of the prosthesis in each image frame. We set two instance of the balloon model: one to get the outer boundary of the prosthesis and another one to get the inner boundary of the prosthesis. In the next stage, we recover the lost part of the bone, following the balloon result in each frame. Finally, the geometry of the prosthesis is reconstructed through a marching cubes technique. In the experimental results we demonstrate the advantages of our technique when compared with the one presented in (You et al., 2009).

\section{PROPOSED METHOD}

The standard format for CT images is DICOM (Digital Imaging and Communications in Medicine). In the case of CT images of the head we find the bone as well as soft tissues (brain, skin, etc.). In a DICOM image, the data matrix has real values in the range $[-1000,1000]$, called CT numbers. It is known that the CT number for bone tissues belongs to the range $[400,1000]$. So, a simple binarization method can be performed in order to highlight just the structure of interest. Besides, we also apply a hole filling method to correct bone topology and a simple search process to discard acquisition artifacts. The Figures 1.(a)-(b) shows the original image and the segmentation result, respectively.

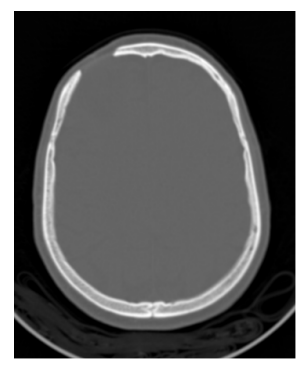

(a)

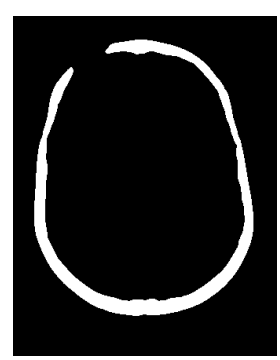

(b)
Figure 1: (a) Slice of the defective skull. (b) Bone segmentation.

Now, once the bone is segmented, we take each frame and get the end points of the lesion and their tangents. In order to perform this task, we apply an edge detector to extract the boundary $B$ of the bone in the binary image and a thinning process to get the skeleton of the bone. The obtained results are pictured on Figure 2.

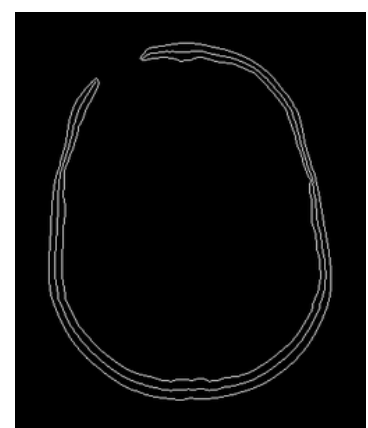

Figure 2: Bone boundary and skeleton.

Then, a convex hull $S$ is computed for the skeleton. We consider the difference $B-S$ as the outer boundary of the bone and the set $B \cap S$ as the in- 
ner boundary of the bone. The obtained curves are used to get the geometric information need for the further step. Firstly we discard a set of boundary points nearby the lost part of the bone, typically 10 points nearby the end points of the skeleton. Then, for each boundary, we take the boundary points inside a neighborhood of each end point to fit a line which direction will be used to get the tangent necessary for the balloon model setup. This process is applied for both, the inner and outer curves, generating the results pictured on Figure 3.

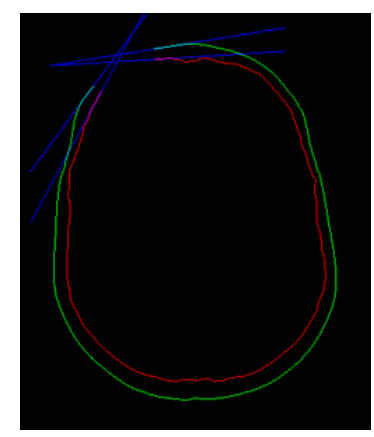

Figure 3: (a) Inner (red) and outer (green) boundaries of the bone plus the tangent lines.

Next, we apply a deformable model, a balloonlike model (Cohen, 1991), to compute the patch of the prosthesis in each image frame. We take the end points and tangents already obtained as boundary conditions for a deformable model. We set two instance of the balloon model: one to get the outer boundary of the prosthesis and another one to get the inner boundary.

Geometrically, the balloon model (Cohen, 1991) is described by a parametric contour $c:[0,1] \rightarrow D \subset$ $\mathfrak{R}^{2}$. From the dynamic viewpoint, we have a deformable contour which is viewed as a time-varying curve $c(s, t)=(x(s, t), y(s, t))$. In this formulation, the Lagrange equations of motion (balloon model equation) can be expressed as:

$$
\dot{c}-\left(\omega_{1} c^{\prime}\right)^{\prime}+\left(\omega_{2} c^{\prime \prime}\right)^{\prime \prime}=\vec{F}_{\text {ext }}+k \vec{n}(c),
$$

subject to $c(0, t), c(1, t), c^{\prime}(0, t), c^{\prime}(1, t)$. In (1) we use the notations $\dot{c} \equiv \frac{\partial c}{\partial t}, c^{\prime} \equiv \frac{\partial c}{\partial s}, c^{\prime \prime} \equiv \frac{\partial^{2} c}{\partial s^{2}}, \vec{n}(c)$ is the unit normal over the curve and $k$ is a scale factor. The field $\vec{F}_{\text {ext }}$ means an external force, which depends on image elements or constraints.

In our case, $\vec{F}_{\text {ext }}=0$, each curve is an open one, fixed in the end points of the lesion following the tangents at that points. Besides these boundary conditions, we must set the initial curve to complete the initialization of each instance of the balloon.

To solve (1) we use finite differences as described in (Cohen, 1991). In order to include the initial curve in the numerical method, we must give an initial sequence of points $C^{0}=c(i \Delta s, 0)=c_{i}^{0}$, with $i=$ $0, \ldots, N$, which is the discrete version of the initial curve. Such curve must satisfies the boundary conditions also. In the case of the outer curve, we can simplify the equations by using the reference system pictured on Figure 4, which has both the end points on the horizontal axis (one in the origin and the other one at $x=L$ ), to write the constraints as:

$$
\begin{aligned}
C(0)=C(L) & =0, \\
C \prime(0) & =\tan \theta_{0}, \\
C \prime(L) & =\tan \theta_{1} .
\end{aligned}
$$

Besides, the initial shape should be as closer as possible to the target to reduce time computation. We observe that a cubic curve, represented by

$$
\begin{aligned}
c(s)= & \left(L s, L\left(\tan \theta_{0}+\tan \theta_{1}\right) s^{3}-\right. \\
& \left.L\left(2 \tan \theta_{0}+\tan \theta_{1}\right) s^{2}+L \tan \theta_{0} s\right),
\end{aligned}
$$

where $s \in[0,1]$, fits all of these requirements.

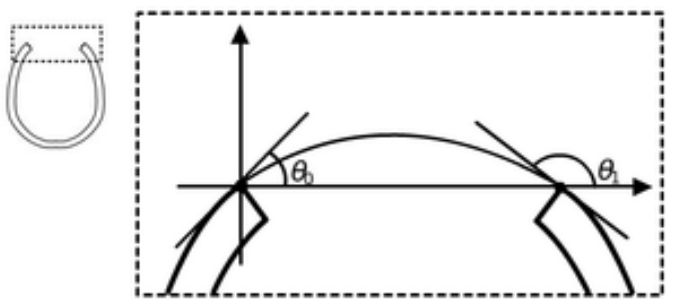

Figure 4: Coordinate system used to setup the outer balloon.

An analogous construction can be made for the inner balloon. The Figure 5.(a) shows a typical result obtained through the deformable model. From this result, we can complete the patch of the prosthesis in the corresponding slice. It is just a matter of filling the region between the obtained curves and take the difference between this region and the bone (Figure 5.(b)). So, by executing this procedure in each slice we can complete the prosthesis volume.

Besides, there are cases in which we observe lost of bone without changing the topology of the corresponding region. The Figure 6.(a) shows such a case. The bone region, pictured in white on Figure 6.(b), has a skeleton given by a closed curve which automatically indicates this problem. In this case, we can assume that the lost region is small. Therefore, we just take the difference between the segmented bone and its convex hull (Figure 6.(c)). Then we discard small islands and the biggest region to isolate the prosthesis patch, pictured on Figure 6.(d). In the final stages, we recover the geometry of the prosthesis through a marching cubes technique. 


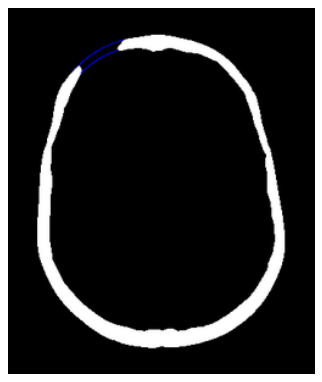

(a)

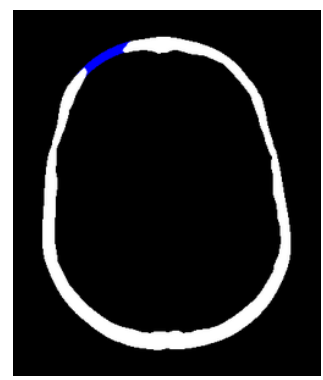

(b)
Figure 5: (a) Balloon results. (b) Prosthesis patch (in blue).

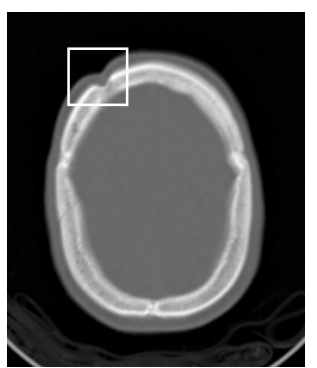

(a)

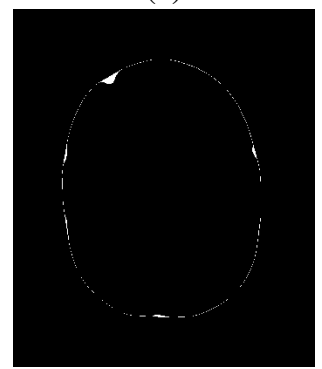

(c)

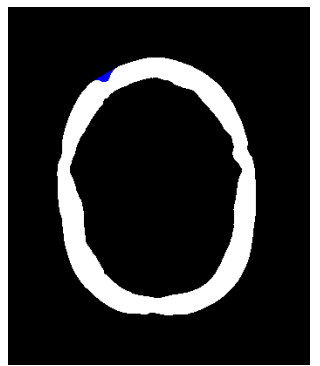

(b)

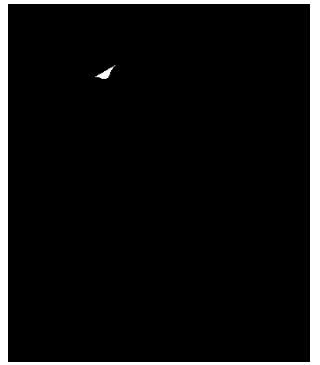

(d)
Figure 6: (a) Original slice with defective region highlighted. (b) Segmented bone. (c) Difference between the convex hull and the bone region. (d) Prosthesis patch.

\section{EXPERIMENTAL RESULTS}

The proposed model is applied to generate the prosthesis for repairing the defective skull pictured on Figure 8.(a). In this case, we can also use a reflection technique, based on the assumption of skull symmetry, to reconstruct the skull. We will use this solution to measure the quality of the result. The defective region intersects 23 slices of the whole image volume (68 slices). Three of them are processed using the convex hull, like in Figure 6 and the other ones are processed through the balloon model.

In the experiments of this section we set null the external force in expression (1). Following (Cohen, 1991), the parameters $\omega_{0}$ and $\omega_{1}$ are set to $(\Delta s)^{2}$ and $(\Delta s)^{4}$, for both the balloon instances where $\Delta s$ is the discretization step applied to get the numerical solution of equation (1). The value of the normal force scale parameter is $k=-0.062$. The stopping criterium for the iterative method used to solve (1) is based on expression $\left\|C^{t+\Delta t}-C^{t}\right\| \leq 10^{-3}$, where $C^{t+\Delta t}$ and $C^{t}$ are the curves at time $t+\Delta t$ and $t$. The Tables 1 and 2 report some statistics about the initialization (expression (3)) and evolution of the balloon instances.

\begin{tabular}{|c|c|c|}
\hline Parameter & Min & Max \\
\hline$\theta_{0}$ & $4^{\circ}$ & $22^{\circ}$ \\
\hline$\theta_{1}$ & $156^{\circ}$ & $169^{\circ}$ \\
\hline No. Interactions & 48 & 135 \\
\hline
\end{tabular}

Table 1: Outer balloon: Statistics for parameters $\left(\theta_{0}, \theta_{1}\right)$ and number of interactions of balloon.

\begin{tabular}{|c|c|c|}
\hline Parameter & Min & Max \\
\hline$\theta_{0}$ & $12^{\circ}$ & $29^{\circ}$ \\
\hline$\theta_{1}$ & $143^{\circ}$ & $156^{\circ}$ \\
\hline No. Interactions & 31 & 147 \\
\hline
\end{tabular}

Table 2: Inner balloon: Statistics for parameters $\left(\theta_{0}, \theta_{1}\right)$ and number of interactions of balloon.

Considering that the mean number of interactions of the numerical scheme is 135 for the outer balloon and 147 for the inner one and we have a mean number of 21 snaxels for the balloon model instance, we can say that the computational cost is not expensive. The Figures 7.(a)-(c) show three slices and the corresponding prosthesis patches. In the first two cases, the result is visually acceptable. However, the Figure 7.(c) shows that the thickness of the prosthesis patch is not suitable in this case, as we can observe with a zoom in the defective region, pictured on Figure 7.(d). This problem happens because we did not get the suitable boundary conditions. We will address this problem in further works.

Figures 8.(b)-(d) show three viewpoints of the repaired skull. A visual inspection indicates that the prosthesis geometry suitably reconstructs the defective region. However, the method is sensitive to the parameters choice. So, we must be careful about this point during the setup of the balloon technique.

In order to measure the quality of the obtained result we compute a similarity measure, based on the distance from the top and inner surfaces of the prosthesis and the prosthesis generated by reflection, taken as a ground truth. Specifically, for each node of the top (inner) surface we calculate the distance from the corresponding surface obtained by reflection. The 


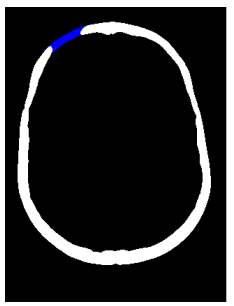

(a)

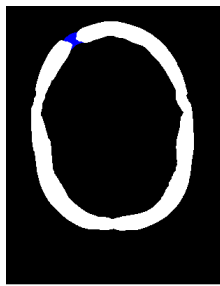

(c)

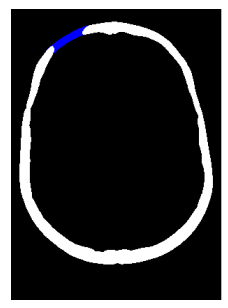

(b)

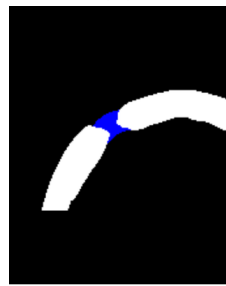

(d)
Figure 7: (a)-(c) Prosthesis patches obtained with our method. (d) Zoom in the patch of last figure.

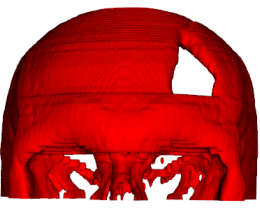

(a)

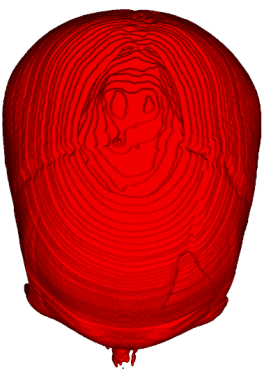

(c)

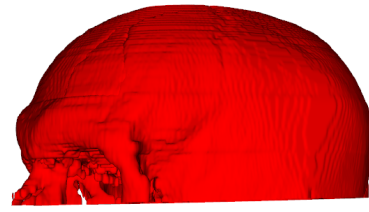

(b)

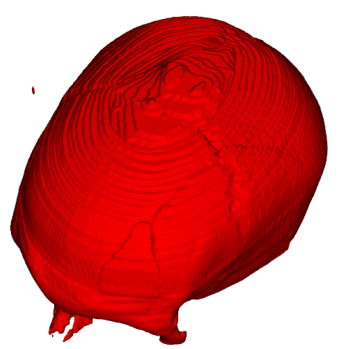

(d)
Figure 8: (a) Original defective skull. (b)-(d) Skull surface reconstructed.

Table 3 reports a statistics for the distance field obtained. For the top surface the reported minimum distance is 0.0 and the maximum one is 2.89 .

Therefore, the distance field lies in the range $[0,2.89]$, which is acceptable if we consider that the resolution of the skull volume is $512 \times 512 \times 68$ voxels. The inner surface precision is smaller; between $[0,3.16]$. However, the Figure 9 shows that the maximum distance happens in small places over the prosthesis surface. This figure shows a color map that pictures the intensity of distance field. This fact is also confirmed through the standard deviation (named StD

\begin{tabular}{|c|c|c|c|}
\hline Distance & Top & Inner & You et al. \\
\hline Min. & 0.0 & 0.0 & 0.06 \\
\hline Max. & 2.89 & 3.16 & 6.06 \\
\hline Mean & 1.10 & 0.72 & 3.09 \\
\hline StD. & 0.52 & 0.48 & 1.34 \\
\hline
\end{tabular}

Table 3: Statistics for the distance field (in voxel units) between the prosthesis surfaces and the ground through (reflection).

in Table 3), which is too smaller then the maximum distance.

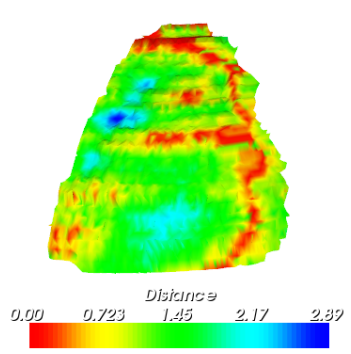

(a)

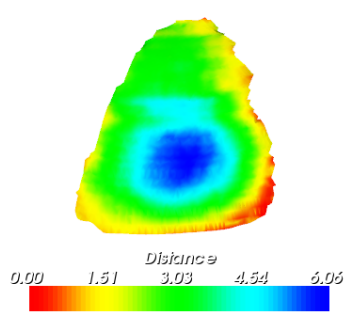

(c)

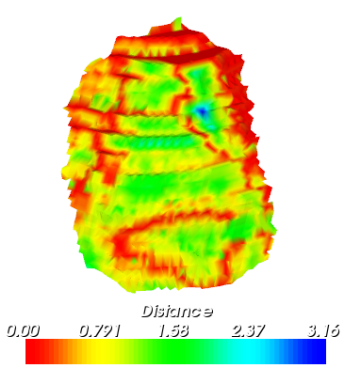

(b)
Figure 9: (a) Distance map plotted over the top surface obtained by our method. (b) Artificial inner surface and colored distance map. (c) Top surface and distance map for You et al. technique.

It is worthwhile to compare our method with the one proposed by You et al. in the reference (You et al., 2009). The Figure 10 pictures the solution obtained with that method. It does not take into account the tangent directions at the boundary of the hole. Therefore, the obtained prosthesis may not fit the curvature of the skull. In fact, the method has a bias towards planar shapes due to the fact that there is no any constraint related to local curvature. We can check this problem in the result pictured on Figure 10 as well as through comparisons with the reflection result presented on the third column of Table 3, for the top surface. All the reported distances are higher than the ones reported by our technique which indicates that we can better recover the curvature of the region. This fact is also confirmed by the distance field pictured on 
Figure 9.(c).

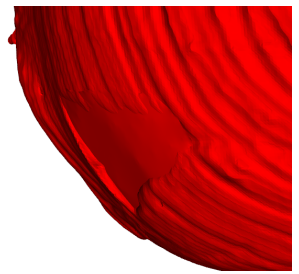

(a)

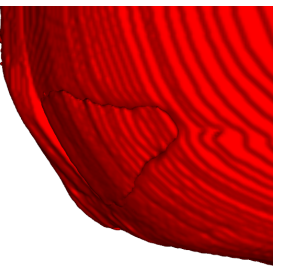

(b)
Figure 10: (a) Viewpoints of the solution obtained by the method described in (You et al., 2009). (b) A viewpoint of the prosthesis generated by our technique.

\section{CONCLUSION AND FUTURE WORKS}

Based on a deformable model, a method is proposed to reconstruct the defective position of a skull. The goal is to construct the prosthesis model for the defective region. We show a promising result and compare our technique with a state-of-the-art one, showing that our method can generate a more suitable prosthesis geometry.

Future directions for our work are to improve the method that obtain the boundary conditions (avoiding the problem pictured on Figure 7). We also plan to test and compare the approach with other available techniques (Hu et al., 2007; Lin et al., 2008). Besides, we intend to apply a $3 D$ deformable model, using DNURBS (Qin and Terzopoulos, 1996), to get the prosthesis geometry. It could address the staircase defect generated by the slice-by-slice strategy and generate more smooth patches.

\section{ACKNOWLEDGMENT}

Authors would like to thank the support provided by CNPq, CAPES (grant 094/2007) and FAPERJ (grant E-26/170.030/2008).

\section{REFERENCES}

Chen, J.-J., Liu, W., Li, M.-Z., and Wang, C.-T. (2006). Digital manufacture of titanium prosthesis for cranioplasty. The International Journal of Advanced Manufacturing Technology, 27(11-12):1148-1152.

Cohen, L. D. (1991). On active contour models and balloons. CVGIP:Image Understanding, 53(2):211-218.
Hu, Q., Yang, H., and Yao, Y. (2007). A software method to model and fabricate the defective bone repair bioscaffold using in tissue engineering. In $\mathrm{Li}, \mathrm{K}$., $\mathrm{Li}, \mathrm{X}$., Irwin, G., and He, G., editors, Life System Modeling and Simulation, volume 4689 of Lecture Notes in Computer Science, pages 445-452. Springer Berlin / Heidelberg.

Kai, C. C., Meng, C. S., Ching, L. S., Hoe, E. K., and Fah, L. K. (2009). Cranioplasty using polymethyl methacrylate prostheses. Journal of Clinical Neuroscience, 16(1):56-63.

Lee, M.-Y., Chang, C.-C., Lin, C.-C., Lo, L.-J., and Chen, Y.-R. (2002). Custom implant design for patients with cranial defects. Engineering in Medicine and Biology Magazine, IEEE, 21(2):38-44.

Lee, S.-C., Wu, C.-T., Lee, S.-T., and Chen, P.-J. (2009). Cranioplasty using polymethyl methacrylate prostheses. Journal of Clinical Neuroscience, 16(1):56-63.

Lin, L., Zhang, J., and Fang, M. (2008). Modelling the bioscaffold for repairing symmetrical and unsymmetrical defective skull. In Bioinformatics and Biomedical Engineering, 2008. ICBBE 2008. The 2nd International Conference on, pages 905-908.

Qin, H. and Terzopoulos, D. (1996). D-nurbs: a physicsbased framework for geometric design. Visualization and Computer Graphics, IEEE Transactions on, 2(1):85 -96.

Sanan, A. M. and Haines, S. J. M. (1997). Repairing holes in the head: A history of cranioplasty. Neurosurgery, 40(3):588-603.

Solaro, P., Pierangeli, E., Pizzoni, C., Boffi, P., Scalese, G., Di Lorenzo, N., and Pirillo, V. (2008). From computerized tomography data processing to rapid manufacturing of custom-made prostheses for cranioplasty: Case report. Journal of Neurosurgical Sciences, 52(4):113-116.

You, F., Hu, Q., Yao, Y., and Lu, Q. (2009). A new modeling method on skull defect repair. Measuring Technology and Mechatronics Automation, 2009. ICMTMA '09. International Conference on, 1(11-12):568-572. 